\title{
Acute encephalopathy with biphasic seizures and late reduced diffusion associated with Epstein-Barr virus
}

\author{
Nobuyuki Nosaka ${ }^{1}$, Yoshinori Ito ${ }^{2 *}$, Shinya Hara ${ }^{1}$ \\ ${ }^{1}$ Department of Pediatrics, Toyota Memorial Hospital, Toyata, Japan; \\ ${ }^{2}$ Department of Pediatrics, Graduate School of Medicine, Nagoya University, Nagoya, Japan. \\ Email: *yoshi-i@med.nagoya-u.ac.jp
}

Received 8 October 2011; revised 12 November 2011; accepted 23 November 2011.

\begin{abstract}
We report the case of a 12 month-old female diagnosed with acute encephalopathy with biphasic seizures and late reduced diffusion. Epstein-Barr virus DNA was detected in both cerebrospinal fluid (511 copies $/ \mathrm{mL}$ ) and in serum ( $828 \mathrm{copies} / \mathrm{mL})$ during the early stage of disease, suggesting a role for this virus in pathogenesis.
\end{abstract}

Keywords: Acute Encephalopathy with Biphasic Seizures and Late Reduced Diffusion; Acute Encephalopathy; Epstein-Barr Virus; MRI

\section{INTRODUCTION}

Studies on acute encephalopathy have progressed rapidly in recent years. The classification of acute encephalopathies has advanced with the discovery of further subtypes based on clinical, radiologic, and laboratory findings [1-4]. Recently, acute encephalopathy with biphasic seizures and late reduced diffusion (AESD) has been described in a cohort of Japanese children [1]. AESD is characterized by a febrile seizure followed within several days by a cluster of secondary seizures and late reduced diffusion on magnetic resonance imaging (MRI). Although the prognosis of patients with AESD was initially reported to be poor, milder forms of AESD without neurologic sequelae have since been recognized $[5,6]$. Several pathogens - for example, influenza virus and human herpesvirus 6 (HHV-6) - have been reported as causative agents of AESD; however, infectious agents have not been detected in all individuals with AESD [1] Infection with other microorganisms may be associated with AESD but go unrecognized as such, due to difficulties in detection of such pathogens.

We report a case of a 12 month-old female with a mild type of AESD concurrent with detection of Epstein-Barr virus (EBV) DNA in serum and cerebrospinal fluid
(CSF). This virus has, until now, not been reported to be associated with AESD.

\section{CASE REPORT}

A 12-month-old, previously healthy, third-generation Japanese-Peruvian female presented to a local emergency department with a self-limited generalized tonicclonic seizure lasting a few minutes. She was on no medication prior to the first seizure and was discharged with a diazepam suppository. The patient remained febrile and slightly drowsy after the first seizure. On day four she was admitted to the hospital with a cluster of seizures. Upon arrival she was alert with a body temperature of $38.8^{\circ} \mathrm{C}$. The seizures were tonic, refractory to typical doses of intravenous diazepam and subsequently required a bolus of intravenous midazolam to be controlled. On examination she displayed mild motor deterioration of the extremities with associated hypotonia, verbal deterioration and inability to visually fixate on objects. Standard laboratory tests showed abnormalities in white blood cell count $(13,200 / \mu \mathrm{L}$; normal, 3900 to $9100 / \mu \mathrm{L})$ without atypical lymphocytes, aspartate transaminase (106 IU/L; normal, 13 to $33 \mathrm{IU} / \mathrm{L})$, alanine transaminase (175 IU/L; normal, 6 to $30 \mathrm{IU} / \mathrm{L})$ and creatinine kinase (1223 IU/L; normal, 62 to $287 \mathrm{IU} / \mathrm{L})$ levels. The blood cell count and levels of protein and sugar in CSF were normal. MRI on admission revealed reduced diffusion in the frontal and parietal lobes bilaterally and in the right temporal lobe (Figure 1(a)). No signal abnormalities were present on the T2-weighted image (Figure 1(b)). An initial electroencephalogram displayed frequent right-dominant frontal spikes. Treatment with phenobarbital and steroid pulse therapy (methylprednisolone, $30 \mathrm{mg} / \mathrm{kg} /$ day for the next three days) was initiated. Psychomotor status gradually improved over the succeeding days. The patient showed improvement in visual pursuit by day eight, was able to hold her head steady by day 10 , and was able to sit without support by day 18 . On day 10 the patient developed a generalized, 

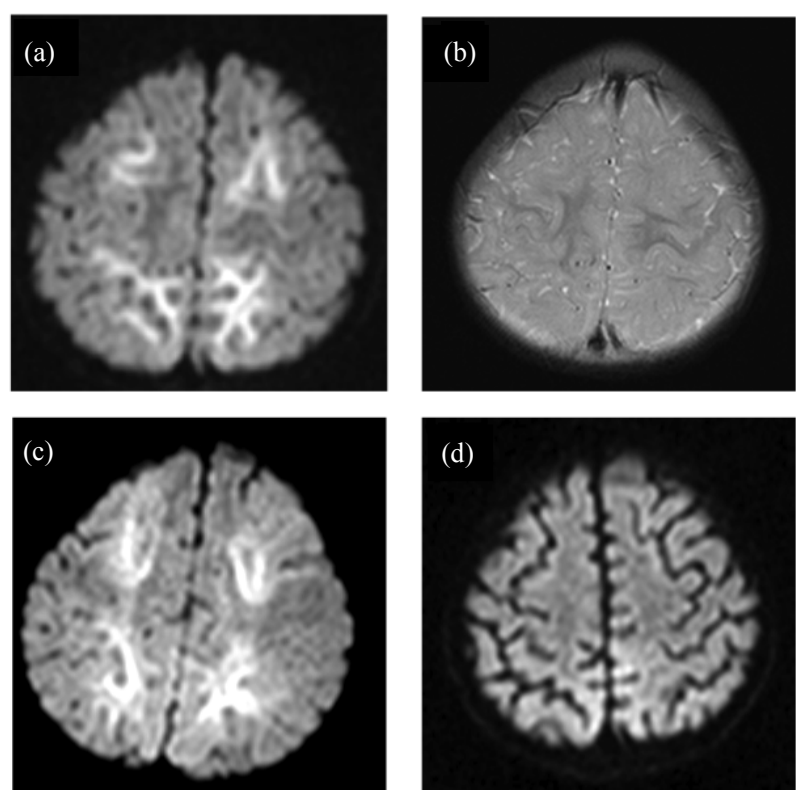

Figure 1. MRI images from a patient with acute encephalopathy with biphasic seizures and late reduced diffusion. (a) Reduced diffusion was observed in the frontal and parietal lobes, bilaterally, on the diffusion-weighted image (DWI) at day 4; (b) T2-weighted image on day 4 showing no signal abnormality; (c) MRI obtained on day 9; the regions that showed abnormalities on the initial MRI displayed higher signals on the DWI; (d) Follow-up DWI image on day 22 showed disappearance of the signal abnormalities without apparent cerebral atrophy.

red, morbilliform eruption without lymphadenopathy. A phenobarbital lymphocyte stimulation test produced a positive result, resulting in a diagnosis of phenobarbital allergy. A repeat MRI was obtained on days 9 and 22 . Regions in which abnormalities were present on the initial MRI displayed higher signals on both diffusion and T2 weighted MRI images on day nine (Figure 1(c)). These abnormalities were no longer present upon MRI on day 22; no cerebral atrophy was observed (Figure 1(d)). The patient's development was normal at 18 months.

Testing for several infectious agents in the patient's serum and CSF was performed by molecular and serologic methods. Levels of EBV DNA in both serum and CSF from the patient were quantified by real-time polymerase chain reaction. EBV DNA was detected at 828 copies $/ \mathrm{mL}$ in serum and 511 copies $/ \mathrm{mL}$ in CSF during the early stage of disease. Serologic tests for anti-EBV antibodies on day five produced the following results: positive for viral capsid antigen $(\mathrm{VCA}) \operatorname{IgG}(\times 320$, immunofluorescence antibody assay; negative, $<10$ ); negative for VCA IgM (negative, $<10$ ) and EBV nuclear antigen (EBNA; negative, $<10$ ) (VCA IgM, negative; VCA $\mathrm{IgG}, \times 320$; EBNA, $\times 10$, in the convalescent period) (Figure 2). HHV-6, HHV-7, VZV, HSV-1 and cytome-

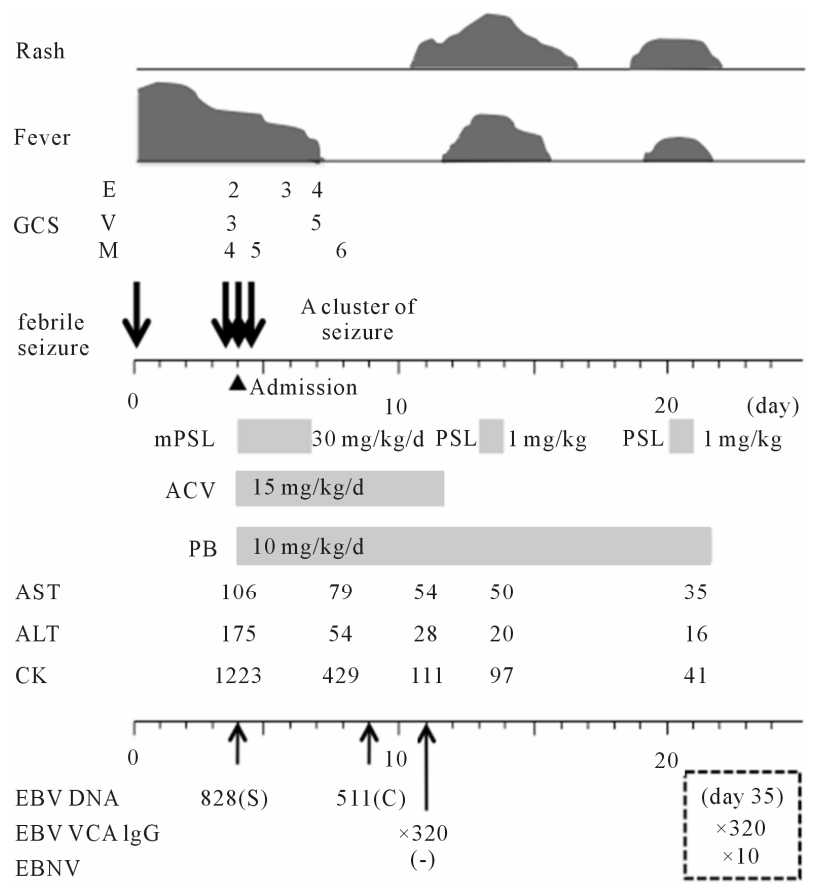

Figure 2. Clinical course of the patient. mPSL, methylprednisolone; PSL, prednisolone; ACV, acyclovir; PB, phenobarbital; EBV, Epstein-Barr virus; VCA, viral capsid antigen; EBNA, Epstein-Barr virus nuclear antigen; $S$, serum; $C$, cerebrospinal fluid.

galovirus DNA were not detected in either CSF or serum. A viral antigen test for influenza virus from a nasopharyngeal swab was negative at the onset of the disease. Serologic tests for viruses other than EBV were positive for HHV-6 IgG $(\times 160$, immunofluorescence antibody assay; negative, $<10$ ) and negative for HHV-6 IgM, influenza A (H1N1, H3N2) and influenza B.

\section{DISCUSSION}

Infectious agents have been previously identified in 10 of 17 reported patients with AESD. The pathogens detected were influenza A and B (four patients), HHV-6 (three patients), HHV-7 (one patient), varicella-zoster virus (one patient), and adenovirus (one patient) [1]. In the case reported here these agents were negative by either serologic tests for viral specific IgM or real-time PCR assay. In contrast, EBV DNA was detected both in CSF (511 copies $/ \mathrm{mL}$ ) and serum ( 828 copies $/ \mathrm{mL}$ ) during the early stage of disease. Serologic assays detected VCA IgG at $\times 320$, but were negative for VCA IgM and EBV nuclear antigen, but were positive for EBNA in the convalescent phase. Although these data are not sufficient to confirm acute EBV infection, the PCR results provide some evidence that EBV infection was associated with AESD in this patient. However, it is also possible that detection of EBV DNA in both CSF and serum resulted from a reactivation of latent EBV infection in 
the context of severe illness.

To our knowledge, this report is the first to describe a patient with AESD associated with EBV infection. EBV is one of the most common identifiable causes of acute childhood encephalitis; EBV has been detected in the central nervous system of $10 \%$ of children with acute encephalitis [7,8]. Neurologic complications of EBV infection are diverse and include encephalitis, cerebellitis, aseptic meningitis, transverse myelitis, GuillainBarre syndrome, cranial neuritis and others [8,9]. EBV encephalitis does not occur in the majority of patients presenting with infectious mononucleosis [9]. MRI has a low specificity for EBV encephalitis [10]. The prognosis associated with EBV encephalitis is controversial and ranges in severity from mild to fatal $[8,9]$. These features were consistent with the present case with the exception of the biphasic clinical course. Because the classic laboratory hallmarks of infectious mononucleosis-for example, typical lymphocytosis - are absent and early diagnosis of EBV infection is sometimes difficult, EBV may be not recognized as a pathogen in cases of AESD. A combination serologic and PCR assay will allow detection of any association of EBV with AESD.

\section{REFERENCES}

[1] Takanashi, J., Oba, H., Barkovich, A.J., et al. (2006) Diffusion MRI abnormalities after prolonged febrile seizures with encephalopathy. Neurology, 66, 1304-1309. doi:10.1212/01.wnl.0000210487.36667.a5

[2] Maegaki, Y., Kondo, A., Okamoto, R., et al. (2006) Clinical characteristics of acute encephalopathy of obscure origin: A biphasic clinical course is a common fea- ture. Neuropediatrics, 37, 269-277.

doi:10.1055/s-2006-955928

[3] Okamoto, R., Fujii, S., Inoue, T., et al. (2006) Biphasic clinical course and early white matter abnormalities may be indicators of neurological sequelae after status epilepticus in children. Neuropediatrics, 37, 32-41. doi:10.1055/s-2006-923949

[4] Yamanouchi, H., Kawaguchi, N., Mori, M., et al. (2006) Acute infantile encephalopathy predominantly affecting the frontal lobes. Pediatric Neurology, 34, 93-100. doi:10.1016/j.pediatrneurol.2005.08.002

[5] Takanashi, J., Tsuji, M., Amemiya, K., Tada, H. and Barkovich, A.J. (2007) Mild influenza encephalopathy with biphasic seizures and late reduced diffusion. Journal of the Neurological Sciences, 256, 86-89. doi:10.1016/j.jns.2007.02.006

[6] Okumura, A., Kidokoro, H., Tsuji, T., et al. (2009) Differences of clinical manifestations according to the patterns of brain lesions in acute encephalopathy with reduced diffusion in the bilateral hemispheres. American Journal of Neuroradiology, 30, 825-830. doi:10.3174/ajnr.A1431

[7] Bale, J.F. (1999) Human herpesviruses and neurological disorders of childhood. Seminars in Pediatric Neurology, 6, 278-287. doi:10.1016/S1071-9091(99)80026-6

[8] Doja, A., Bitnun, A., Ford Jones, E.L., et al. (2006) Pediatric Epstein-Barr virus-associated encephalitis: 10-Year review. Journal of Child Neurology, 21, 384-391.

[9] Domachowske, J.B., Cunningham, C.K. and Cummings, D.L. (1996) Acute manifestations and neurologic sequelae of Epstein-Barr virus encephalitis in children. Pediatric Infectious Disease Journal, 15, 871-875. doi:10.1097/00006454-199610000-00008

[10] Shian, W.J. and Chi, C.S. (1996) Epstein-Barr virus encephalitis and encephalomyelitis: MR findings. Pediatric Radiology, 26, 690-693. doi:10.1007/BF01356839 